V.2, N.1, 2018

DOI: $10.33947 / 2595-6264-v 2 n 1-3673$

\title{
A INDÚSTRIA 4.0 E SUAS CONTRIBUIÇÕES À SUSTENTABILIDADE
}

\author{
INDUSTRY 4.0 AND ITS CONTRIBUTIONS TO SUSTAINABILITY
}

\author{
Bianca C. Sanches ${ }^{1}$, Emily S. Carvalho², Fabio Fonseca Barbosa Gomes ${ }^{3}$
}

\begin{abstract}
RESUMO
O presente artigo relata como a Indústria 4.0 apresentando-se como a nova revolução industrial e os impactos causados com a implantação das tecnologias na questão da sustentabilidade, um assunto que tem se tornado cada vez mais preocupante. A Indústria 4.0 cumpre seu papel na sustentabilidade com contribuições econômicas, ambientais e sociais em toda sua cadeia de valor. Foram apresentadas as formas que trarão benefícios e que aumentarão as condições de sustentabilidade, como a eficiência na utilização de recursos, a interação da logística e eficiência social e ambiental. Além disso, essas mudanças também dependem da mudança de hábito da população, como a reutilização de recursos descartáveis, a geração de menos lixo e uma maior ação na área de reciclagem, para permitir que a sociedade tenha um ambiente mais organizado e de mais qualidade.
\end{abstract}

PALAVRAS-CHAVE: Indústria 4.0. Sustentabilidade. Ambiental.

\section{ABSTRACT}

This paper reports how Industry 4.0 presents itself as the new industrial revolution and the impacts caused by the implantation of technologies in the question of sustainability, a subject that has become increasingly worrying. Industry 4.0 fulfills its role in sustainability with economic, environmental and social contributions throughout its value chain. It was presented the forms that will bring benefits and that will increase the conditions of sustainability, such as the efficiency in the use of resources, the interaction of logistics and social and environmental efficiency. In addition, these changes also depend on changing the population's habit, such as the reuse of disposable resources, the generation of less waste and more action in the area of recycling, to allow society to have a more organized and more quality environment.

KEYWORDS: Industry 4.0. Sustainability. Environmental.

\footnotetext{
Graduanda da UNINASSAU

Graduanda da UNINASSAU

Possui mestrado em Sistemas e Computação pela Universidade Salvador (2016), especialização em Redes de Computadores e Telecomunicações pela Universidade Salvador (2010) e graduação em Bacharelado em Sistemas de Informação pelo Centro Universitário Estácio da Bahia (2007). Atualmente é professor mediador a distância no Instituto Federal da Bahia - Lauro de Freitas, professor mestre nas instituições de ensino superior Centro Universitário Regional do Brasil e Centro Universitário Dom Pedro II. Tem experiência na área de Ciência da Computação, com ênfase em Redes de Computadores, atuando principalmente nos seguintes temas: computação ubíqua e pervasiva, automação residencial, educação, sistemas de informação e redes de computadores.
} 


\section{INTRODUÇÃO}

A barreira entre virtual e físico ou máquina e ser humano, tem sido transposta em um ritmo cada vez mais acelerado. Isto é resultado da produção industrial, que é responsável por movimentar o curso da sociedade, portanto acompanha esse progresso. Assim, em 2011, na Alemanha, surgiram as primeiras ideias sobre a Indústria 4.0, apresentando-se como a próxima revolução industrial e oferecendo fábricas, produtos e serviços inteligentes (RIBEIRO, 2017).

Em contraponto a todo esse progresso tecnológico, o planeta vive a maior crise ecológica de toda a história (AMBERGER et al., 2010). O uso inconsequente dos recursos naturais faz a humanidade beirar o esgotamento dos mesmos e o desgaste de estruturas essenciais à vida na Terra, como a camada de ozônio e os ecossistemas. Tomando consciência de tamanho risco, o conceito de sustentabilidade tem se tornado cada vez mais popular, pois o mesmo busca um estilo de vida em que o progresso não conflite com a vida do planeta.

$O$ desafio da sustentabilidade precisa ser implantado, para exercer um impacto real na diminuição e consequentemente eliminação dos índices de detrimento ambiental. Para isto, as propostas de inovação da indústria 4.0 apresentam-se como uma das principais aliadas a esse objetivo. Devido ao seu pioneirismo, a Alemanha lidera o ranking de pesquisas e publicações acadêmicas relacionadas ao tema, sendo seguida pelos Estados Unidos e China, e o resultado desses materiais são iniciativas como o projeto Advanced Manufacturing Partnership e o plano Made in China 2025, que visam iniciar o processo de integração entre tecnologia e indústria em seus respectivos países (NETO et al., 2018).

O Brasil, todavia, não se encontra entre os 10 países com mais publicações, mantendo-o na retaguarda desse importante marco socioeconômico e tecnológico e na solução de diversos problemas ambientais, mesmo sendo um dos mais ricos territórios ecológicos (figura 1).

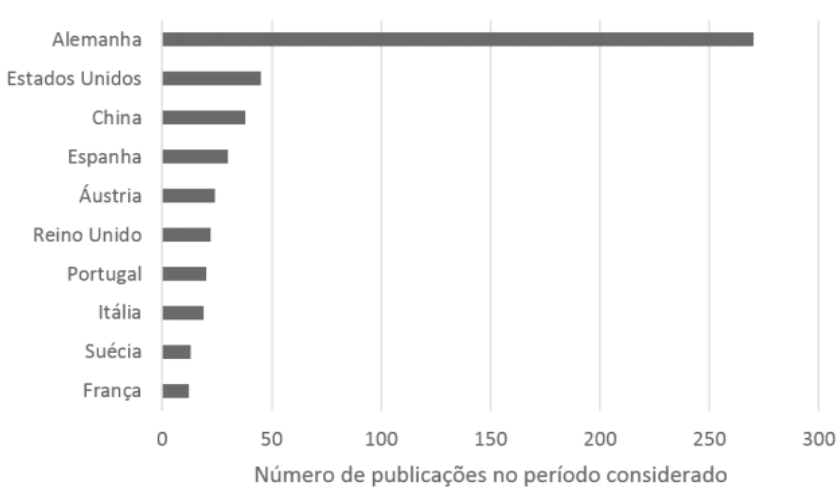

Figura 1. Número de publicações por país (Neto et al., 2018)

O objetivo do trabalho é apresentar os impactos positivos e a importância da indústria 4.0 para um futuro mais sustentável. Para isso, este artigo irá explorar o desenvolvimento da quarta revolução industrial e seus conceitos, abordando crises ambientais da atualidade e, também, as contribuições das mudanças industriais na área de sustentabilidade. A metodologia utilizada neste estudo foi a pesquisa bibliográfica exploratória, desenvolvida a partir dos impactos causados com a implantação das tecnologias da Indústria 4.0 na questão da sustentabilidade, um assunto de preocupação e discussão mundial.

\section{A QUARTA REVOLUÇÃO INDUSTRIAL}

Apesar do conceito da quarta revolução industrial também estimular a reindustrialização e a competitividade industrial, ele tem como principais alvos: (i) a eficiência energética e de recursos, (ii) a redução dos ciclos de time-to-market (tempo de mercado), bem como o (iii) aumento da produtividade.

Sendo assim, a Indústria 4.0 é um complexo conjunto de diversas de tecnologias que permitem que as máquinas e os humanos trabalhem de maneira colaborativa. Este conjunto de tecnologias é compreendido por tecnologias de manufatura avançada, big data, Internet das Coisas e sistemas cyber-físicos, que juntos tornam linhas de produção ágeis e o atendimento ao consumidor final personalizado. 


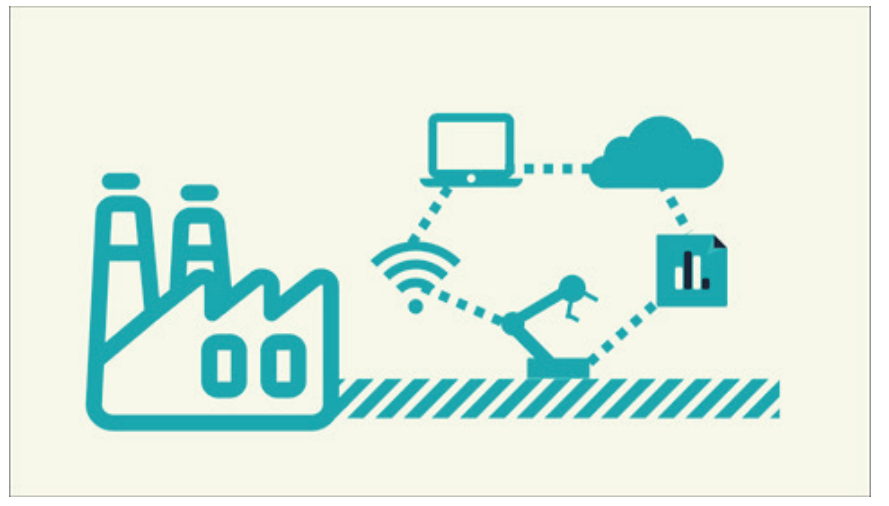

Figura 2 - As tecnologias habilitadoras.

Tais tecnologias foram sendo agregadas ao conceito para possibilitar a utilização da orientação a serviço, a alteração dos processos produtivos em tempo real com a tomada de decisões mais seguras dos sistemas cyberfísicos, modularidade, interoperabilidade e virtualização do sistema de produção.

A produção em um sistema cyber-físico depende de uma rede que interligue a tecnologia da informação, os componentes mecânicos e eletrônicos das máquinas, pois as informações geradas nesta rede, são capazes de criar mudanças na atuação das empresas. (RIBEIRO, 2017)

Em relação a tecnologia de sistemas cyber-físicos, as mudanças a indústria vem sendo realizadas através de integração de sistemas de maneira vertical, que permite uma visão global do funcionamento da empresa, e de maneira horizontal, que acompanha o ciclo de vida do produto, trazendo benefícios como redução de energia e do tempo de produção e logística, e também o aumento da qualidade dos produtos (STOCK, SELIER, 2016) .

A indústria 4.0 tem seguido modelo RAMI 4.0 (Reference Architectural Model for Industry 4.0 oi Modelo de Referência Arquitetural para a Indústria 4.0) para padronizar a arquitetura e comunicação que fazem esses sistemas funcionarem, ordenando a informação em camadas, desde a fabricação até a gestão, conforme pode ser visto através da figura 3.

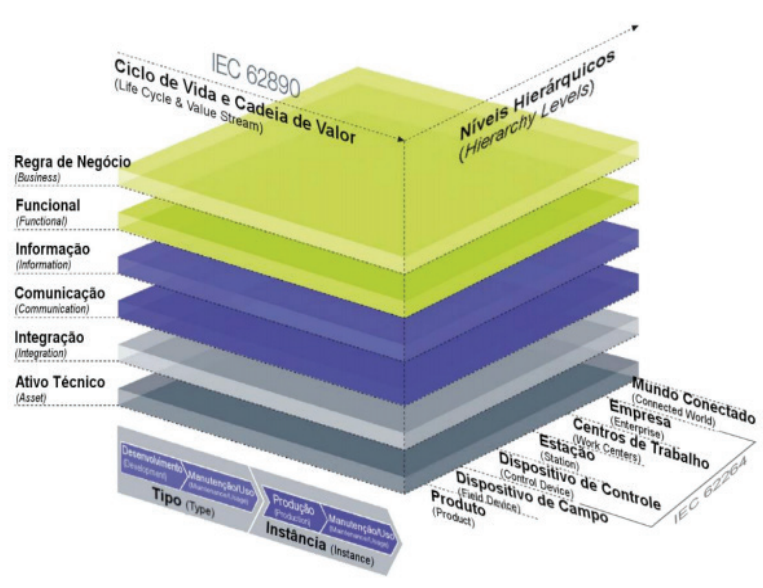

Figura 3 - RAMI (Adophs et al., 2015)

De acordo com a figura 3, pode-se ver que a estrutura do RAMI é dividida em níveis que podem ser subdivididos em: (i) Ativo Técnico, (ii) Integração, (iii) Comunicação, 9iv) Informação, (v) Funcional e (vi) Regra de Negócio. Cada uma das camadas tem características específicas para o bom funcionamento do RAMI.

Além disso, ela pode ser dividida através de Níveis hierárquicos, que são (i) Produto, (Dispositivo de Campo), (iii) Dispositivo de Controle, (iv) Estação, (v) Centros de Trabalho, (vi) Empresa e, finalmente, (vii) Mundo Conectado. Todas as camadas do RAMI são interligadas entre si e tem como o objetivo o funcionamento adequado para fazer com que a Indústria 4.0 funcione de forma eficiente.

\subsection{INTERNET DAS COISAS}

Levando a conexão à internet além dos computadores, a Internet da Coisas torna possível controlar objetos remotamente ou torná-los provedores de recursos. Para que sejam capazes de realizar processamento os objetos precisam de microcontroladores e processadores e utilizar uma linguagem comum a múltiplos dispositivos. É necessário também sensores que coletam dados referentes aos objetos, enviam-nos para armazenamento utilizando redes cabeadas, Wi-Fi ou bluetooth para comunicação (KAGERMAN et al, 2013).

$\mathrm{Na}$ indústria, essa comunicação recebe o nome de Internet Industrial das Coisas (IolT - Internet of Industrial Things) que cria uma rede inteligente entre equipamentos, sistemas e indivíduos em toda a empresa, pelo ciclo de vida inteiro do produto. 


\section{V.2, N.1, 2018}

\subsection{BIG DATA}

Big Data é o termo que se refere ao grande volume de informação oriundo da internet assim como a capacidade de processar e qualificar o que pode ser relevante. Para esse processamento existe o Big Data Analytics, que reúne métodos e técnicas para mineração e gestão de dados consubstanciando conhecimentos relevantes e proveitosos, como por exemplo a inteligência artificial (HERMANN, 2016).

O Big Data e a Inteligência Artificial se aplicam na Indústria 4.0 mediante o uso de algoritmos de mineração de dados, que são capazes de produzir estatísticas e previsões precisas com o que é coletado e analisado dos dispositivos e sensores, bases históricas de interrupção de serviços e alertas de manutenção preventiva. O resultado desse processo é a redução de defeitos, na otimização da matéria-prima e da energia elétrica (WANG, 2016).

\subsection{COMPUTAÇÃO EM NUVEM}

Sendo fonte de recursos computacionais e o acesso sob demanda via internet, a computação em nuvem viabiliza a variação do número de servidores e espaço de armazenamento de acordo com o fluxo de demanda, otimizando custos e operando de forma dinâmica e ágil.

É a computação em nuvem que possibilita que todos os dados gerados pelos elementos que compõem a empresa, sejam armazenados, tratados e protegidos, para servirem à análise dos dados, tão como o uso de sistemas de controle de acesso às informações (KAGERMAN et al, 2013).

Por exemplo, a indústria pode utilizar um serviço de virtualização que permita construir, implementar e compartilhar soluções de análise em tempo real da operação de um parque fabril. $E$ por meio de armazenamento e serviços na nuvem, a indústria pode aplicar algoritmos de inteligência artificial para auxiliar na análise preditiva (WANG, 2016). Com base nesses dados, a indústria poderá tomar decisões mais assertivas quanto aos recursos materiais e humanos, etapas e volume de produção, períodos de manutenção etc. visando redução de custos e aumento de produtividade.

\subsection{MANUFATURA AVANÇADA}

No setor industrial, a associação entre robótica e elementos de outras tecnologias, como robôs controlados via internet e que além de executar suas atividades for- necem informações à linha de produção, deu origem a robótica avançada e tem maximizado a qualidade dos produtos e serviços, a saúde e segurança dos funcionários e redução do consumo de energia e de recursos. A conectividade fornecida pela internet das coisas torna os robôs aptos a se conectar à sistemas e comunicarse com outras máquinas e ainda trabalhar colaborativamente com os seres humanos (AZEVEDO, 2017).

Outra protagonista da nova revolução industrial é a manufatura aditiva, processo de sobreposição de material para criar objetos baseando-se em um modelo virtual tridimensional, geralmente a adição da matéria-prima ocorre em camada sobre camada, ou contrário da usinagem, que aplica a técnica de manufatura por remoção de material. Causando mudanças também na indústria a manufatura digital, projeta tridimensionalmente o processo de produção em desenhos assistidos por computador (CAD), representando todos os elementos envolvidos em uma simulação virtual, permitindo análise e correção de eventuais problemas sem a montagem real da linha produtiva, apontando melhorias e otimização da manufatura atual (PRAUSE; ATARI, 2017).

\section{CRISE AMBIENTAL E SUSTENTABILIDADE}

A crise ambiental é um assunto de discussão mundial que envolve diversas partes sociais, muito $A$ crise é um somatório de ações, que representa a busca humana desesperada por desenvolvimento. São diversos problemas que foram surgindo com o decorrer do tempo, sendo difícil distinguir qual seria o mais grave, sendo que um problema interfere no outro de forma direta e indireta.

A sociedade vive em um mundo superlotado, "nós vivemos num planeta em que, em menos de 200 anos, saltamos de 1,5 bilhão de pessoas para 6,5 bilhões. Em 40 anos, deveremos ser 9 bilhões na Terra. Precisamos entender que não estamos enfrentando um problema pequeno, como escassez de petróleo: viveremos o fenômeno de que tudo será escasso" (AMBERGER et al., 2010). O nível de consumo atualmente no mundo, principalmente das classes mais altas, gera uma exploração que passa dos limites na Terra, tornando a pegada ecológica insustentável.

A Sustentabilidade é um conjunto de ações realizadas por pessoas que visam atender as necessidades atuais dos seres humanos, sem comprometer o futuro das próximas gerações, portanto podemos imaginar que o desenvolvimento econômico está ligado diretamente 


\section{V.2, N.1, 2018}

com o termo, pois é preciso desenvolver economicamente, mas sem agredir o meio ambiente.

Seguem algumas ações sustentáveis:

a) Exploração dos recursos vegetais de florestas e matas de forma controlada, garantindo o replantio sempre que necessário;

b) Preservação total de áreas verdes não destinadas a exploração econômica;

c) Ações que visem o incentivo à produção e consumo de alimentos orgânicos, pois estes não agridem a natureza além de serem benéficos à saúde dos seres humanos;

d) Exploração dos recursos minerais (petróleo, carvão, minérios) de forma controlada, racionalizada e com planejamento;

e) Uso de fontes de energia limpas e renováveis (eólica, geotérmica e hidráulica) para diminuir o consumo de combustíveis fósseis. Esta ação, além de preservar as reservas de recursos minerais, visa diminuir a poluição do ar;

f) Criação de atitudes pessoais e empresarias voltadas para a reciclagem de resíduos sólidos. Esta ação além de gerar renda e diminuir a quantidade de lixo no solo, possibilita a diminuição da retirada de recursos minerais do solo;

g) Desenvolvimento da gestão sustentável nas empresas para diminuir o desperdício de matéria-prima e desenvolvimento de produtos com baixo consumo de energia;

h) Atitudes voltadas para o consumo controlado de água, evitando ao máximo o desperdício. Adoção de medidas que visem a não poluição dos recursos hídricos, assim como a despoluição daqueles que se encontram poluídos ou contaminados.

A área de Tecnologia da Informação (TI) nas empresas tem papel muito importante para a estratégia. Utilizando os recursos tecnológicos existentes, as empresas conseguem disponibilizar produtos ou serviços cada vez mais sofisticados para seus clientes fazendo a diferença no mercado, mas para isso é preciso estar preparada tecnologicamente em termos de computadores, servidores, infraestrutura, climatização.

Para Preusler et al. (2015), todo o aparato tecnológico precisa estar alinhado com o custo para manter o funcionamento e sem agredir o meio ambiente. É a partir daí que surge o conceito TI Verde, portanto as empresas precisam se desenvolver tecnologicamente, mas sem agredir o meio ambiente adotando práticas sustentáveis como:

a) Redução de consumo de papel nas empresas;

b) Redução com cartuchos e tones de impressoras;

c) Descarte correto do lixo eletrônico;

d) Virtualização de servidores, a fim de reduzir o consumo de energia em datacenters;

e) Aumento do uso da computação em nuvem;

f) Reciclagem do lixo eletrônico.

Segundo Elkington (1997), o desenvolvimento sustentável do tripé da Sustentabilidade ou Triple Bottom Line, que é o resultado da confluência entre as áreas: social, econômica e ambiental, conforme pode ser visto na figura 4 .

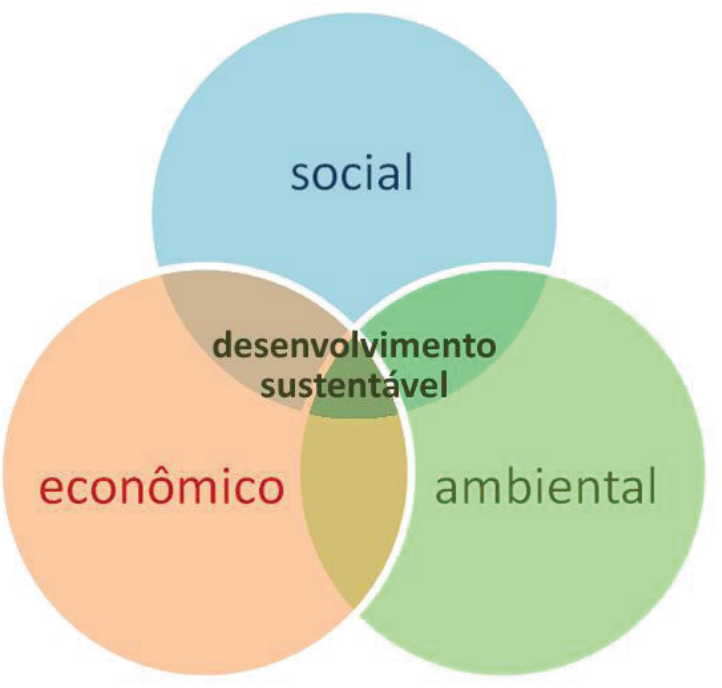

Figura 4 - Tripé da Sustentabilidade (ELKINGTON, 1997)

Pode-se ver, analisando a figura 4, que é impossível ter desenvolvimento sustentável com apenas dois elementos. Os critérios Econômico, Ambiental e Social unidos são capazes de formar desenvolvimento sustentável. Desta forma, percebe-se que a adoção de ações de sustentabilidade garante a médio e longo prazo um retorno financeiro, mas o compromisso com a sustentabilidade garante uma imagem diferenciada das empresas, além de um maior interesse por parte de investidores (ELCKINGTON, 1997).

Esse modelo também pode ser aplicado com sucesso na administração pública de forma bastante eficiente. Além disso, os autores afirmaram que "...é possível praticar soluções que permeiem a sustentabilidade, as- 


\section{V.2, N.1, 2018}

segurando a conservação e a preservação ambiental" (VENTURINI e LOPES, 2015).

Esses são os valores centrais das empresas que promovem práticas sustentáveis: redução de custos, conservação de energia e recursos, consumo de energia renovável, criação de normas e condições no trabalho, responsabilidade social nos produtos $\mathrm{e}$ serviços, minimização de embalagens e redução de emissão de carbono.

\section{A INDÚSTRIA 4.0 NO DESENVOLVIMENTO DA SUSTENTABILIDADE}

Além da otimização do uso da matéria-prima proporcionada pela manufatura aditiva, a produção mais assertiva e sob demanda habilitada pelas demais tecnologias envolvidas no processo, a indústria 4.0 cumpre o seu papel na sustentabilidade com contribuições econômicas, ambientais e sociais em toda sua cadeia de valor.

O design de produtos concentra-se na realização de ciclos de vida fechados para produtos, permitindo a reutilização e remanufatura do produto específico, utilizando princípios cradle-to-cradle, conceito que modela a indústria humana nos processos da natureza sendo os materiais como nutrientes circulantes. Este design é possível com a aplicação de sistemas de identificação, para recuperar os núcleos para remanufatura, ou aplicando-os em novos serviços (STOCK, 2016).

Segundo Stock (2016), o retrofitting, que consiste em adicionar um componente ou acessório a um equipamento que não o possuía quando foi fabricado, permite de forma econômica atualizar equipamentos de fabricação existentes com sistemas de sensores e atuadores, bem como com as lógicas de controle relacionadas.

O Retrofitting pode ser usado para a realização de um CPS através de um módulo de criação de valor, como uma fábrica, com equipamentos de fabricação já existentes. Estende a fase de uso ou facilita a aplicação em uma nova fase de uso para o equipamento de fabricação e pode contribuir essencialmente para as dimensões econômica e ambiental da sustentabilidade.

\subsection{CASOS DE SUCESSO COM O USO DA INDÚSTRIA 4.0}

Existem alguns casos de sucesso com o uso da tecnologia 4.0 no Brasil, um dos exemplos é apresentado pelos autores Mendes et al. (2017), em que foi realizada uma pesquisa em uma empresa automobilística que uti- lizou a Indústria 4.0 em seu chão de fábrica. O uso desta tecnologia resultou em uma diminuição dos custos da fábrica e, consequentemente, aumentou a competitividade para a produção de veículos.

As tecnologias utilizadas para a produção de veículos são:

a) TAGS RFID: A produção dos veículos é realizada através de uma TAG RFID que identifica cada um dos veículos durante a produção.

b) Medição inteligente: os veículos têm as suas medições feitas através do uso da tecnologia de laser para verificar se todas as peças do veículo estão em conformidade com o que foi planejado

c) Logística inteligente, com o uso do Automatic Guide Vehicle ou Guia Automático do Veículo, em que o automóvel avança por um transportador de materiais, tudo controlado por um computador de bordo. Outra opção seria o uso de uma guia flexível

d) Fábrica Digital: "A Fábrica Digital utiliza programas computacionais interligados que simulam virtualmente os processos produtivos antes de suas implantações" (MENDES et al.,2017). Uma das grandes vantagens desta fábrica é que as situações e erros podem ser simulados antes de irem para a produção real, detectando possíveis erros (figura 5).

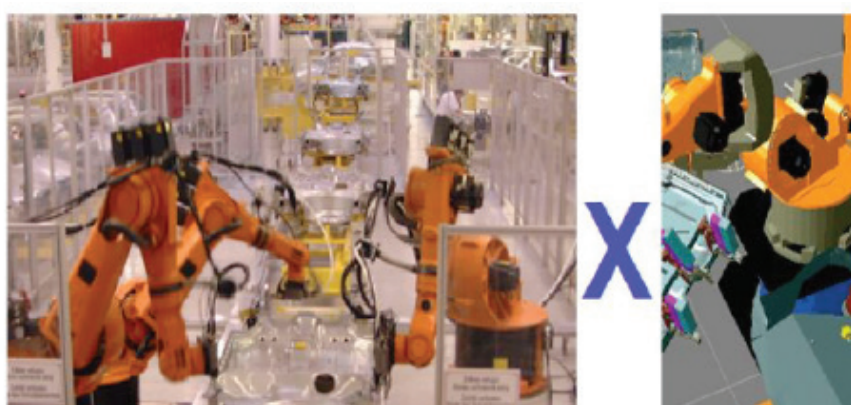

Figura 5 - Fábrica Digital (MENDES et al., 2017)

e) Impressão 3D: Utilização da impressão 3D para imprimir peças em que os engenheiros irão trabalhar para a realização de testes e verificar a compatibilidade da peça.

Para Mentes et al. (2017), as soluções apresentadas na empresa de automóveis mostraram-se bastante vantajosa e ela conseguiu atingir bons resultados com o uso da indústria 4.0 .

Outro caso que merece ser citado é o de Veríssimo e Loureiro (2018), que apresenta algumas oportunidades 
de aplicação da Indústria 4.0 em uma usina de etanol fictícia chamada Ethan LS.

Neste trabalho, os autores simularam situações em que a indústria 4.0 poderia ser aplicada. As tecnologias apresentadas nesta simulação envolvem as tecnologias base de uma fábrica inteligente, Internet das Coisas, Big Data, CPS e Computação em Nuvem utilizando a tecnologia de rede sem fio RFID para rastreio da frota de caminhões da empresa (figura 6).

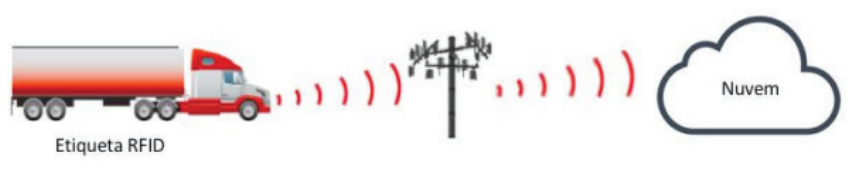

Figura 6 - Uso da tecnologia RFID para rastreio de caminhões (VERÍSSIMO e LOUREIRO, 2018)

Como forma de análise, os autores criaram uma variação percentual teórica (desconsiderando os custos de implementação das tecnologias) de 15\% de aumento na receita e $16 \%$ de redução de custo ao final de 1 ano após a digitalização dos processos. Desta forma, foi possível quantificar os resultados da transformação digital na empresa. Os resultados apontaram uma redução de $17,03 \%$ do custo total. Além disso as receitas aumentaram em $22,80 \%$, demonstrando o sucesso da indústria 4.0

\section{CONSIDERAÇÕES FINAIS}

Neste estudo observou-se como a Industria 4.0 foi se apresenta como a nova revolução industrial. Além disso, através dos casos de sucesso vistos neste artigo, percebe-se que esta tecnologia irá trazer uma grande quantidade de benefícios, valorizando ainda mais as condições de Sustentabilidade. Como resultado disso, percebe-se um aumento na eficiência da utilização de recursos e, também, a interação da logística e eficiência social e ambiental.

O uso desta tecnologia também tem como objetivo mostrar que é possível utilizar de forma eficiente os recursos da natureza e, a partir disso, trazer mais eficiência e eficácia na produção de produtos. Para trabalhos futuros pretende-se aplicar as tecnologias da indústria 4.0 em uma empresa que ainda não possui esta tecnologia e analisar os resultados.

\section{REFERÊNCIAS}

ADOLPHS, P.; BEDEMBENDER, H.; DIRZUS, D.; EHLICH, M.; EPPLE, U.; HANKEL, M.; HEIDEL, R.; HOFFMEISTER, M.; HUHLE, H.; KÄRCHER, B.; KOZIOLEK, H.; PICHLER, R., POLLMEIER, S.; SCHEWE, F.; WALTER, A.; WASER, B.; WOLLSCHLAEGER, M.. Reference Architecture Model Industrie 4.0 (RAMI 4.0) and Zvei, 2015. Disponível em: https://www.zvei.org/en/ subjects/industry-4-0/the-reference-architectural-model -rami-40-and-the-industrie-40-component/. Acesso em: mar. 2019.

AZEVEDO, M.T. Transformação digital na indústria: indústria 4.0 e a rede de água inteligente no Brasil. 2017. 177 p. Escola Politécnica da Universidade de São Paulo, São Paulo, 2017.

ELKINGTON, John. Cannibals with Forks: the triple bottom line of 21st Century Business, Oxford, Capstone, 1997.

HERMANN, M., PENTEK, T.; OTTO, B. Design principles for industrie 4.0 scenarios. In: System Sciences (HICSS), 2016 49th Hawaii International Conference on. IEEE,. p. 3928-3937, 2016.

KAGERMANN, Henning; WAHLSTER, Wolfgang; HELBIG, Johannes (Ed.). Recommendations for implementing the strategic initiative industrie 4.0: final report of the Industrie 4.0 working group. [S.I.]: Forschungsunion, Acatec, 2013.

MENDES, C.; FRANZ, B. S.; CAMPOS, M. M. Estudos de caso da indústria 4.0 aplicados em uma empresa automobilística. POSGERE. São Paulo. n. 1. p. 15-25, 2017.

NETO, Anis Assad; PEREIRA, Gustavo Bernadi; DROZDA, Fabiano Oscar; SANTOS, Adriana de Paula Lacerda. A busca de uma identidade para a indústria 4.0. Braz. J. of Develop., Curitiba, v. 4, n. 4, p. 13791395, 2018.

PRAUSE, G.; ATARI, S. 2017. In sustainable production networks for Industry 4.0, Entrepreneurship and Sustainability Issues 4(4): 421-431.

RIBEIRO, Joaquim Meireles. $O$ conceito da Indústria 4.0 na confecção: análise e implementação. Universidade do Minho. Escola de Engenharia, 2017.

STEHL, J. P. V.; LOUREIRO, R. B. A. Engenharia Química no contexto da indústria 4.0: estudo de caso em uma usina de Etanol. Niterói: UFF, 2018. 
V.2, N.1, 2018

STOCK, T., SELIGER, G. Opportunities of Sustainable Manufacturing in Industry 4.0, Procedia CIRP, v. 40, 2016, p. 536-541, ISSN 2212-8271.

PREUSLER, Taísa Scariot; CRESPI, Tatiane Baseggio; NARDI, Valdeci; MARAFON, Daiane Lampugne. Terceirização dos serviços de impressão como forma de obtenção da ecoeficiência em uma empresa pública de pesquisa agropecuária. In: SINGEP - Simpósio Internacional de Gestão de Projetos, Inovação e Sustentabilidade, IV, 2015, São Paulo, pp. 1-10.
VENTURINI, Lauren Dal Ben; LOPES, Luis Felipe Dias. O modelo Triple Bottom Line e a sustentabilidade na administração pública: pequenas práticas que fazem a diferença. UFSM, 2015.

WANG, S et al. Implementing Smart Factory of Industrie 4.0: an outlook. International Journal of Distributed Sensor Networks. Guangzhou, 2016. 